\title{
Inflammation and oxidative stress markers in diabetes and hypertension
}

\author{
Chloé Pouvreau' \\ Antoine Dayre' \\ Eugene G Butkowski \\ Beverlie de Jong ${ }^{2}$ \\ Herbert F Jelinek ${ }^{2,3}$ \\ 'Faculty of Sciences, University of \\ Poitiers, Poitiers, France; ${ }^{2}$ School of \\ Community Health, Charles Sturt \\ University, Albury, NSW, Australia; \\ ${ }^{3}$ Australian School of Advanced \\ Medicine, Macquarie University, \\ Sydney, NSW, Australia
}

This article was published in the following Dove Press journal: Journal of Inflammation Research

\begin{abstract}
Background: Inflammation and oxidative stress are important factors associated with chronic disease such as essential hypertension (HTN) and type 2 diabetes mellitus (T2DM). However, the association of inflammation and oxidative stress in HTN with T2DM as a comorbidity is inconclusive due to the multifactorial nature of these cardiometabolic diseases.

Methodology: The influence of pathophysiological factors include genetics, age of patient, and disease progression change throughout the lifespan and require further investigation. The study population included 256 participants attending a rural health screening program who were tested for markers of inflammation, oxidative stress, and coagulation/fibrinolysis. Demographic and clinical variables included, age, gender, systolic and diastolic blood pressures, blood glucose, hemoglobin A1c, estimated glomerular filtration rate, and cholesterol profile. Data were tested for normality, and nonparametric statistics were applied to analyze the sample with significance set at $p<0.05$. Results: Of the inflammatory markers, interleukin-1 $\beta$ (IL-1 $1 \beta$ ) and IL-10 were significantly different between the control and hypertensive group $(p<0.03)$ and between the HTN+T2DM compared to the HTN group $(p<0.05)$. Significant results for oxidative stress were observed for urinary 8-iso$\mathrm{PGF}_{2} \alpha$ and insulin-like growth factor 1 (IGF-1) between the control and the HTN+T2DM group $(p<0.01)$. Glutathione $(\mathrm{GSH})$ was also significant between the HTN and HTN+T2DM group $(p<0.05)$. Investigation of the progression of $\mathrm{HTN}$ also found significant changes in the inflammatory markers IGF-1, monocyte chemoattractant protein 1 (MCP-1), and (MCP-1/IGF-1)*IL-6 $(p<0.05)$. Conclusion: This study demonstrated that 8 -iso- $\mathrm{PGF}_{2} \alpha$ and erythrocyte GSH may be clinically useful for assessing HTN and HTN with T2DM as a comorbidity, while significant changes in the inflammatory profile were also observed with HTN progression.
\end{abstract}

Keywords: hypertension, type 2 diabetes mellitus, inflammation, oxidative stress, biomarkers

\section{Introduction}

Hypertension (HTN) is a public health problem which affects over 1 billion people world-wide. ${ }^{1}$ HTN is often associated with type 2 diabetes mellitus (T2DM), approximately $75 \%$ of people with T2DM will develop HTN. ${ }^{2}$ These findings raise major health issues as long-term HTN increases the risk of coronary heart disease, stroke, heart failure, and peripheral vascular disease, ${ }^{3}$ and diabetes associated with HTN is known to increase cardiovascular disease (CVD) risk by up to threefold. ${ }^{4}$

Obesity, inflammation, oxidative stress, and insulin resistance are part of a common pathophysiological pathway for HTN and T2DM. Studies have confirmed that oxidative stress and increased inflammatory processes often co-exist, and are considered to be common causes and risk factors for T2DM and HTN. However, the link between oxidative stress and inflammatory processes is inconclusive, requiring more mechanistic
Correspondence: Eugene G Butkowski School of Community Health, Charles Sturt University, Elizabeth Mitchell Drive, Albury, NSW 2640, Australia

Tel +6I 2605 I 9337

Email ebutkowski@csu.edu.au 
studies that highlight the interaction between these processes and how they affect diabetes, HTN, and when HTN is found in the presence of T2DM. ${ }^{5,6}$

Hyperglycemia and arterial stiffness increase with age and affect endothelial function as part of HTN and T2DM pathophysiology. ${ }^{7}$ The autonomic nervous system also plays a role in HTN and T2DM, with sympathetic nervous system dysfunction linked to hyperinsulinemia and increased blood pressure (BP) by increasing cardiac output. ${ }^{8}$ Diabetes and HTN disease progression leads to changes in endothelial dysfunction and increased pro-inflammatory and oxidative stress markers and a decrease in antioxidant and anti-inflammatory biomarkers. $^{9-12}$ These inflammatory and oxidative stress changes can lead to atherosclerosis, accelerated development of arterial thrombosis, and increased risk of death due to CVD. ${ }^{13,14}$

Oxidative stress occurs due to an imbalance between ROS formation resulting in enhanced ROS generation and an imbalance in the production of antioxidants. ${ }^{15}$ Optimum levels of the body's antioxidant reserve are affected by hyperglycemia as found in diabetes and HTN. ${ }^{11,16}$ HTN has been proposed to be associated with an increase in oxidative stress. ${ }^{17}$ Diabetes and associated hyperglycemia affects the redox state and is reflected in decreased levels of reduced glutathione (GSH) and an increase in oxidized glutathione (GSSG). ${ }^{13} 8-\mathrm{OHdG}$, an endothelial cell oxidative stress marker is a product of DNA base modification produced by the oxidation of deoxyguanosine. Increasing urinary 8-OHdG excretion has been observed in prediabetes and T2DM compared with healthy subjects and is considered to be a useful marker for the detection of early micro- and macrovascular complications in T2DM. ${ }^{18}$ In HTN patients with T2DM increased urinary 8-OHdG excretion has been demonstrated. ${ }^{19}$ Similarly, isoprostanes, which are stable products generated in vivo by peroxidation of arachidonic acid due to free radical activity, are useful markers for oxidative stress. Urinary excretion of 8 -iso- $\mathrm{PGF}_{2} \alpha$ has been found to be higher in hypertensive and diabetic patients when compared to healthy controls. ${ }^{16,20}$ The increase in urinary 8 -iso- $\mathrm{PGF}_{2} \alpha$ is associated with elevation of serum total cholesterol (TC) and reduction of high-density lipoprotein cholesterol (HDL-C) that can lead to atherosclerosis and coronary heart disease. ${ }^{20}$ Hypertensive and normotensive diabetic patients have similar urinary 8 -iso- $\mathrm{PGF}_{2} \alpha$ excretion, suggesting similar mechanisms with respect to glycemic control, lipid peroxidation, and antioxidation mechanisms. ${ }^{21,22}$ Atherosclerosis is associated with HTN and is characterized by oxidative stress and pro-inflammatory activity that may be associated with the observed imbalance between coagulation and fibrinolysis. ${ }^{23}$ Markers of coagulation and fibrinolysis include D-dimer and
C5a. Previous studies have shown an increase in the plasma levels of C5a and D-dimer in prediabetic patients, ${ }^{11}$ and the increased coagulation may be due to increased inflammation but in turn can also enhance inflammation. ${ }^{24,25}$

Increased coagulation and oxidative stress result in an increase in pro-inflammatory cytokines such as interleukin-1 $\beta$ (IL-1 $\beta$ ), IL-6, and monocyte chemoattractant protein 1 (MCP-1), and a decrease in insulin-like growth factor I (IGF-1) in the plasma of hypertensive and T2DM patients. ${ }^{12,26-28}$ Higher levels of IL-6 have been reported to be associated with obesity and insulin resistance and have been demonstrated in macrovascular complications in T2DM patients. ${ }^{12}$ The increased production of IL-1 $\beta$ in human pancreatic $\beta$-cells is induced by high glucose concentration affecting insulin secretion and decreases cell proliferation and apoptosis. ${ }^{29}$ However, the anti-inflammatory cytokine IL-10, plays a role in the regulation of the innate immune system and inhibits the production of pro-inflammatory cytokines in T2DM and limits BP in HTN. ${ }^{30,31}$ Some studies have recognized the benefits of increasing IGF-1 in HTN and diabetes. Higher plasma IGF-1 bioavailability may protect against the development of TD2M and vascular complications associated with HTN. ${ }^{32}$ However, the association between IGF-1 and HTN is complex, depending on the concentration of IGF-1 as it stimulates IL-10 production. ${ }^{33,34}$ Levels of the pro-inflammatory marker MCP-1 in turn depends on the levels of IGF-1 and IL-1. When IGF-1 is low or IL-1 is elevated, MCP-1 increases and is involved in the recruitment of monocytes and macrophages into the vasculature, as is the case in HTN and T2DM. Higher levels of MCP-1 are associated with increased CVD mortality. ${ }^{35,36}$

This study therefore aimed to increase our understanding of the impact and interaction of inflammatory and oxidative stress biomarkers in contributing to the pathophysiology of HTN with and without T2DM within a clinical setting. This further understanding may assist in efforts to assist diagnosis, prevention, and treatment of HTN in the presence of diabetes.

\section{Methodology}

The study was approved by the Charles Sturt University Ethics in the Human Research Committee (Ethics approval number: 2006/042) and followed the Helsinki protocol. All participants provided written informed consent to take part in this study. Data for this study were obtained from a total of 256 participants (female:male, 157:99) attending the Charles Sturt University rural health screening clinic. Inclusion and exclusion criteria were kept to a minimum to reflect routine patient presentation to general practice in a rural population. 
Exclusion criteria were CVD, kidney disease, any evidence of acute inflammation, and any evidence of depressive illness. All participants were assessed for waist circumference (WC), $\mathrm{BP}$, and body mass index (BMI). Fasting blood glucose (FBG), hemoglobin A1c (HbAlc), lipid profiles including TC, triglycerides (TG), HDL-C, low-density lipoprotein cholesterol (LDL-C), TC/HDL-C ratio, and D-dimer were performed by the local National Association of Testing Authorities accredited pathology laboratory. Markers of oxidative stress and inflammation were also analyzed. A screening FBG was determined for each participant using the Accu-Chek ${ }^{\circledR}$ system (Hoffman-La Roche Ltd., Basel, Switzerland).

Participants were divided into three groups: a control group, a hypertensive group (HTN), and a hypertensive with diabetes group (HTN+T2DM). The participants were also divided into stage 1 and stage 2 HTN with and without the presence of T2DM. In accordance with the American Diabetes Association, diabetes was determined as a blood glucose level of $\geq 7$ $\mathrm{mmol} / \mathrm{L} .{ }^{37} \mathrm{HTN}$ was defined as a BP $\geq 140 \mathrm{mmHg}$ (systolic blood pressure [SBP]) and/or $\geq 90 \mathrm{mmHg}$ (diastolic blood pressure $[\mathrm{DBP}])$ according to the criteria of the Australian Heart Foundation. ${ }^{38}$ Stage 1 and stage 2 hypertensive participants were classified according to the American Hypertension Guidelines. ${ }^{39}$

$\mathrm{BP}$ was measured with participants in a supine position after a 5 min rest. Brachial artery BP was recorded using a Welsh-Allyn BP recorder (Welsh-Allyn, Sydney, Australia). Weight was measured in $\mathrm{kg}$ using portable scales, without footwear and with only light clothes. Height was measured in $\mathrm{m}$ with participants barefoot and standing with their feet together. WC was measured in $\mathrm{cm}$. BMI was defined as weight in $\mathrm{kg}$ per height in m squared. ${ }^{40}$

The measurement of oxidative stress and DNA damage was assessed by measuring urinary 8 -iso- $\mathrm{PGF}_{2} \alpha, 8 \mathrm{OHdG}$. GSH/GSSG was assayed on erythrocyte lysates.

8-iso- $\mathrm{PGF}_{2} \alpha$ was assessed using the OxiSelectTM 8-isoProstaglandin F $2 \alpha$ ELISA Kits (Cell BioLabs, Inc, San Diego, CA, USA).This assay incorporates a competitive binding ELISA strategy, allowing the 8 -iso- $\mathrm{PGF}_{2} \alpha$ contained in samples and standards to compete with 8 -iso- $\mathrm{PGF}_{2} \alpha-\mathrm{HRP}$ conjugate for binding to an anti-8-iso- $\mathrm{PGF}_{2} \alpha$ antibody fixed to a goat anti-rabbit antibody pre-coated microplate. $8-\mathrm{OHdG}$ was determined using the OxiSelect ${ }^{\mathrm{TM}}$ Oxidative DNA Damage ELISA Kit 8-OHdG Quantitation (Cell BioLabs, INC). 8-OHdG contained in samples and standards competes with an HRP conjugate secondary antibody for binding to an 8-OHdG/BSA conjugate coated microplate. Erythrocyte reduced GSH and GSSG was determined using the Cayman ${ }^{\mathrm{TM}}$ Total Glutathione (GSSG/GSH) Assay Kit. Erythrocyte lysates were prepared by adding $4 \times$ volume of ice-cold $5 \%$ metaphosphoric acid to washed red cells and centrifuged to obtain the red cell lysate. The assay procedure incorporated GSH reductase to reduce reducing GSSG to GSH in the presence of NADPH. GSSG was measured by subtraction after the addition of 2 vinyl pyridine.

Plasma IL-6, IL-1 $\beta$, IL-10, MCP-1, and IGF-1 levels were determined using ELISA provided by Elisakit.com ${ }^{\mathrm{TM}}$ (Jomar Pty Ltd, Melbourne, Australia). All inflammatory markers utilized the same analytical principle. Standards and samples are bound to the specific antibody pre-coated on a microplate. A biotin-conjugated anti-human biomarker antibody was added which binds to human biomarker captured by the antibody. After addition of streptavidin-HRP and substrate solution, color development was measured according to the assay protocol. Plasma C5a was determined using Human C5a Platinum ELISA $^{\mathrm{TM}}$ (eBioscience, Affymetrix, San Diego, CA, USA). All ELISA measurements conducted on the inflammatory, oxidative stress biomarkers were carried out with a Thermo Scientific Multiskan FC ${ }^{\mathrm{TM}}$ (Thermo Fisher Scientific, Waltham, MA, USA). Data analysis for the ELISA analysis utilized 4-parameter logistic curve fit software.

Statistical analysis was performed with SPSS V20 (IBM Corporation, Armonk, NY, USA). To determine if there were significant differences in biomarker levels between the different groups for non-normally distributed data, the non-parametric Kruskal-Wallis test for multiple groups was applied followed by the Mann-Whitney $U$-test as a post hoc test. Anthropometric data were analyzed using ANOVA followed by the Fisher's least significant difference post hoc test. A $p$-value $\leq 0.05$ was considered significant. Results in Tables $1-3$ are expressed as mean \pm 1 SD.

\section{Results}

Anthropometric and general biochemistry data are shown in Table 1. Participants were comparable for age and gender except for the control group, which presented with a lower age. Medication showed some differences between groups as expected, since the control group was medication free and the HTN only group were not on any diabetic medication. Of note is that a large proportion of the T2DM participants were using statins $(75.9 \%)$ in addition to antidiabetic medication. The majority of HTN participants reported use of antihypertensive medication. Use of non-steroidal antiinflammatory drugs (NSAID) was lower in controls than HTN and HTN+T2DM groups.

No significant difference in FBG levels was observed between control and the HTN group. Blood glucose level 
however was significantly increased in the HTN+T2DM group $(p<0.05)$. A significant difference between the control and HTN group and the control and HTN+T2DM was observed in BMI, WC, HbA1c, SBP, DBP, and in antihypertensive medication use $(p<0.05)$. Blood glucose level, BMI, HbA1c, TC, HDL-C, LDL-C, TC/HDL-C ratio, and medication usage differed significantly between the HTN and HTN+T2DM group $(p<0.05)$ (Table 1$)$.

Findings for oxidative stress and inflammatory markers are shown in Figure 1 and Tables 2 and 3. A slight reduction in C5a levels in the HTN group compared to the control group was observed but the difference was not significant $(p=0.06)$. Similarly D-dimer was trending upwards in the HTN and HTN+T2DM group but was also not significant $(p=0.065)$ compared to control.

Of the oxidative stress markers, urinary 8 -iso- $\mathrm{PGF}_{2} \alpha$ increased significantly from control levels to levels observed in the T2DM+HTN group ( $p=0.009)$. The increase observed for 8-iso- $\mathrm{PGF}_{2} \alpha$ levels for the HTN group to the T2DM+HTN group approached significance with $p=0.059$. Reduced GSH was significantly lower in TD2M+HTN compared to control ( $p=0.091)$ and similarly significantly lower than the HTN group, $p=0.032$ (Figure 1).

Analysis of the plasma inflammation markers in the three groups revealed a significant decrease in IL-1 $\beta$ levels in the control compared to the HTN group ( $p=0.017)$, but returned to control levels in the HTN+T2DM group, which was also significantly different to control $(p=0.044)$. The anti-inflammatory cytokine IL-10 decreased significantly from control to the HTN levels $(p=0.03)$ and approached control levels in the HTN+T2DM group (Table 2). Plasma IGF-1 levels decreased from the control group to the HTN group but were only significant between control and the HTN+T2DM group $(p=0.007)$. Several inflammatory ratios, which generally mirror physiological processes such as IL-6/IL-10 were also proposed and analyzed for significance between the three groups. The results, although not significant, did show a trend highlighting their potential usefulness in differentiating between control, HTN, and HTN+T2DM.

Hypertensive participants were further divided into HTN subgroups according to international guidelines as stage 1 HTN with SBP between 140 and $159 \mathrm{mmHg}$ and DBP $\leq 99$ $\mathrm{mmHg}$, stage $2 \mathrm{HTN}$ with SBP $\geq 160 \mathrm{mmHg}$ and/or DBP $\geq$ $100 \mathrm{mmHg}$ with or without T2DM. ${ }^{41}$ Table 2 includes inflammatory biomarkers demonstrating significant differences between stages of HTN.

Plasma IGF-1 levels decreased significantly between the control group and stage $1 \mathrm{HTN}+\mathrm{T} 2 \mathrm{DM}(p=0.002)$ as well as stage 1 HTN and HTN+T2DM ( $p=0.006)$. Stage 2 HTN had the lowest IGF-1 levels but was not significant compared to stage 1 HTN. A significant increase in MCP-1 level was observed

Table I Characteristics of the subjects included in this study

\begin{tabular}{|c|c|c|c|}
\hline Category & Control & HTN & HTN+T2DM \\
\hline Group (N) & 81 & 88 & 87 \\
\hline Gender (female) \% & 61.7 & 63.6 & 58.6 \\
\hline Age (years) & $64.9 \pm 10.5^{\mathrm{a}, \mathrm{b}}$ & $69.9 \pm 9.0$ & $70 \pm 10.5$ \\
\hline \multicolumn{4}{|l|}{ Medication (\%) } \\
\hline Dmeds & $0^{\mathrm{b}}$ & $0^{c}$ & 83.9 \\
\hline Antihypertensive & $0^{\mathrm{a}, \mathrm{b}}$ & $68.2^{c}$ & 87.4 \\
\hline Statins & $13.6^{b}$ & $21.6^{c}$ & 75.9 \\
\hline NSAID & $25.9^{\mathrm{a}, \mathrm{b}}$ & 48.9 & 55.2 \\
\hline Glucose (mmol/L) & $5.3 \pm 0.7^{b}$ & $5.3 \pm 0.6^{c}$ & $8.3 \pm 3.5$ \\
\hline BMI $\left(\mathrm{kg} / \mathrm{m}^{2}\right)$ & $25.7 \pm 4 .\left.\right|^{\mathrm{a}, \mathrm{b}}$ & $28.1 \pm 5.0^{c}$ & $30.3 \pm 6.1$ \\
\hline \multicolumn{4}{|l|}{$W C(\mathrm{~cm})$} \\
\hline Female & $88 \pm 10.1^{a, b}$ & $94.8 \pm 18.5^{c}$ & $99 \pm 14.3$ \\
\hline Male & $97.4 \pm 9.7^{b}$ & $102.2 \pm 10.7^{c}$ & $108.2 \pm 14.6$ \\
\hline Systolic BP (mmHg) & $119.7 \pm 11.2^{\mathrm{a}, \mathrm{b}}$ & $138.5 \pm 18.5$ & $137.5 \pm 18.4$ \\
\hline Diastolic BP $(\mathrm{mmH})$ & $73.6 \pm 6.4^{\mathrm{a}, \mathrm{b}}$ & $78.9 \pm 7.6$ & $77.4 \pm 9.6$ \\
\hline $\mathrm{HbAlc}(\%)$ & $5.6 \pm 0.3^{b}$ & $5.7 \pm 0.3^{c}$ & $6.7 \pm 0.8$ \\
\hline $\mathrm{TC}(\mathrm{mmol} / \mathrm{L})$ & $5.3 \pm 0.8^{b}$ & $5.6 \pm I^{c}$ & $4.5 \pm 1.2$ \\
\hline Triglyceride $(\mathrm{mmol} / \mathrm{L})$ & $1.1 \pm 0.6^{b}$ & $1.3 \pm 0.6^{c}$ & $1.8 \pm 1.2$ \\
\hline HDL-C (mmol/L) & $1.7 \pm 0.4^{b}$ & $1.6 \pm 0.5^{c}$ & $1.4 \pm 0.6$ \\
\hline LDL-C (mmol/L) & $3.1 \pm 0.7^{b}$ & $3.3 \pm I^{c}$ & $2.2 \pm 0.9$ \\
\hline TC/HDL-C ratio & $3.3 \pm 1^{\mathrm{a}}$ & $3.7 \pm 1.1$ & $3.5 \pm 1.4$ \\
\hline
\end{tabular}

Notes: ${ }^{a}$ Significant difference between control and HTN group $(p<0.05)$; ${ }^{b}$ significant difference between control and HTN+T2DM group ( $\left.p<0.05\right)$; ${ }^{c}$ significant difference between HTN and HTN+T2DM group $(p<0.05)$.

Abbreviations: BP, blood pressure; Dmeds, diabetes medication; HbAlc, hemoglobin AIc; HDL-C, high-density lipoprotein cholesterol; HTN, hypertension; LDL-C, lowdensity lipoprotein cholesterol; NSAID, non-steroidal anti-inflammatory drugs; T2DM, type 2 diabetes mellitus; TC, total cholesterol; WC, waist circumference. 


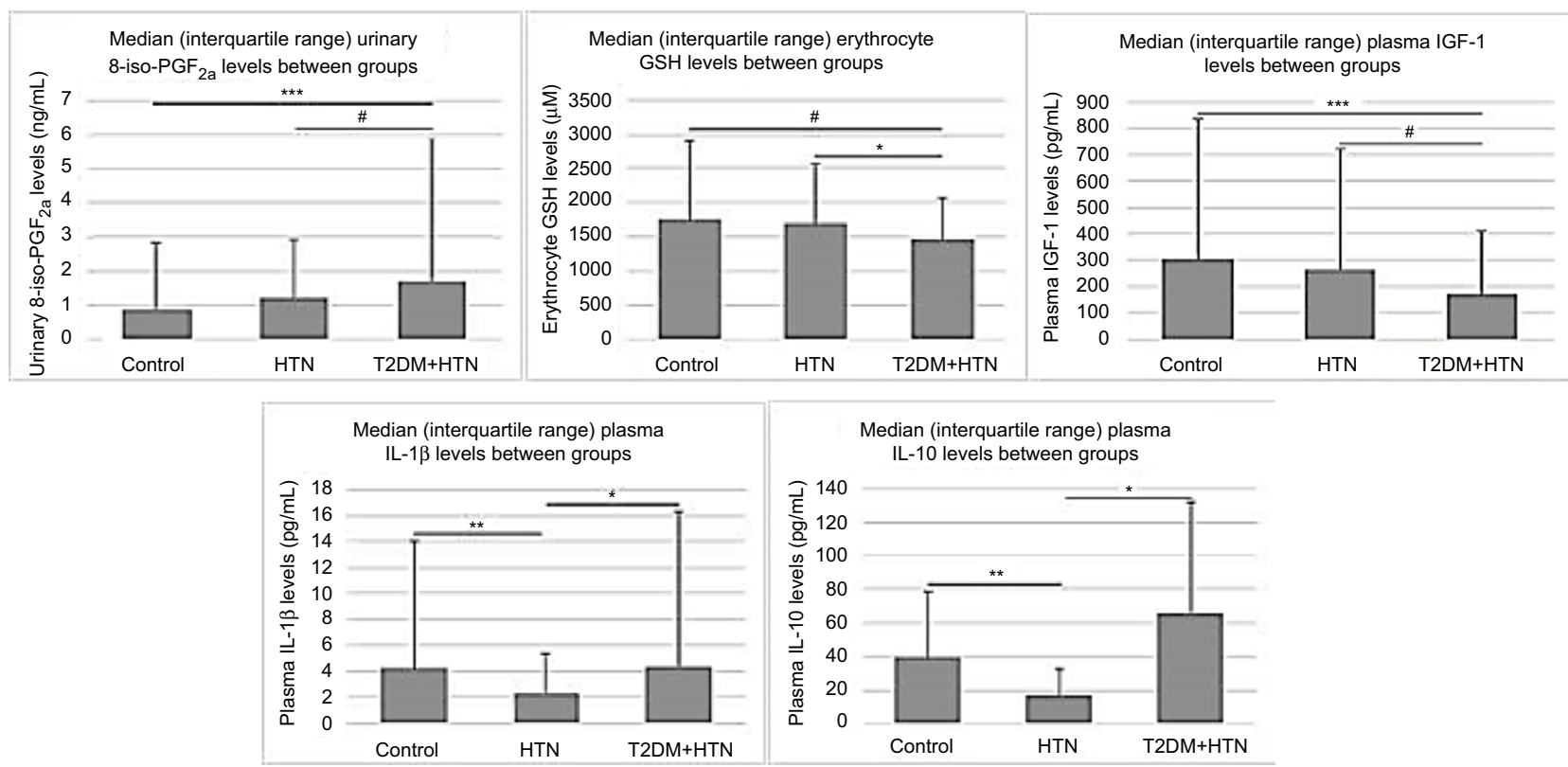

Figure I Impact of hypertension and diabetes on biomarker levels.

Notes: ${ }^{*} p<0.1, * p<0.05, * * p<0.03, * * * p<0.01$.

Abbreviations: HTN, hypertension; IGF I, insulin-like growth factor; IL-I $\beta$, interleukin-I $\beta$; T2DM, type 2 diabetes mellitus.

Table 2 Biomarker levels for control, hypertension, and hypertension with T2DM

\begin{tabular}{|c|c|c|c|}
\hline Category & Control & HTN & HTN+T2DM \\
\hline C5A (ng/mL) & $30.8(24.9) n=66$ & $20.8(27.4) n=74$ & $22.5(33.9) n=67$ \\
\hline D-DIMER $(\mu \mathrm{g} / \mathrm{mL})$ & $0.39(0.44) n=48$ & $0.45(0.35) n=53$ & $0.39(0.29) n=5 I$ \\
\hline 8-ISO-PGF- ${ }_{2} \alpha(\mathrm{ng} / \mathrm{mL})$ & $0.89(1.93)^{b} \mathrm{n}=75$ & $1.22(\mathrm{I} .70) \mathrm{n}=84$ & $1.74(4.15) n=82$ \\
\hline 8-OHDG (ng/mL) & I58.1 (135.8) $n=62$ & $157.3(140.2) n=62$ & $145 . \mid(|6| .3) n=54$ \\
\hline $\mathrm{GSH}(\mu \mathrm{M} / \mathrm{L})$ & $1762(1 \mid 49) n=37$ & $1705(878)^{c} n=40$ & $1457(600) n=27$ \\
\hline GSSG $(\mu \mathrm{M} / \mathrm{L})$ & $286(152) n=37$ & $384(2 \mid 4) n=40$ & $277(250) n=27$ \\
\hline GSH/GSSG & $6.3(6.2) n=37$ & $6.1(6.2) n=40$ & $4.4(4.6) n=27$ \\
\hline $\mathrm{IL}-\mathrm{I} \beta(\mathrm{pg} / \mathrm{mL})$ & $4.2(9.8)^{a} n=53$ & $2.4(3.0)^{c} n=52$ & $4.3(12.0) n=66$ \\
\hline IL-6 (pg/mL) & $14.3(15.3) n=78$ & $16.8(22.8) n=80$ & $17.7(30.7) n=72$ \\
\hline IL-I0 (pg/mL) & $21.5(39.1)^{\mathrm{a}} \mathrm{n}=54$ & $14.5(15.9)^{c} n=53$ & $17.7(65.5) n=66$ \\
\hline IL-IB/IL-I0 & $0.16(0.21) n=53$ & $0.18(0.3 \mathrm{I}) \mathrm{n}=52$ & $0.15(0.53) n=66$ \\
\hline IL-6/IL-10 & $0.41(1.28) n=53$ & $0.58(1.32) n=53$ & $0.42(0.77) n=54$ \\
\hline IGF-I (pg/mL) & $303(533)^{\mathrm{b}} \mathrm{n}=53$ & $266(454) n=54$ & $169(240) n=66$ \\
\hline MCP-I (pg/mL) & $187(80) n=54$ & $193(113) n=53$ & $198(137) n=66$ \\
\hline (MCP-I/IGF-I)*IL-IB & $3.9(8.9) n=53$ & $2.0(4.4) n=52$ & $2.8(11.1) n=65$ \\
\hline$($ MCP-I/IGF-I)*IL-6 & $7.3(19.2) n=52$ & $9.5(45.4) n=53$ & II.I (34.I) $n=54$ \\
\hline
\end{tabular}

Notes: aSignificant difference between control group and HTN group ( $p<0.03)$; bsignificant difference between control group and T2DM with HTN group ( $p<0.01)$;

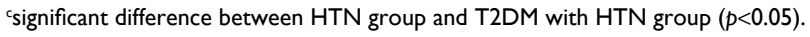

Abbreviations: HTN, hypertension; IGF I, insulin-like growth factor; IL-I $\beta$, interleukin-I $\beta$; MCP-I, monocyte chemoattractant protein I; T2DM, type 2 diabetes mellitus.

between stage 1 and control and between stage 1 and stage 2 HTN groups $(p<0.05)$. The ratio of $(\mathrm{MCP}-1 / \mathrm{IGF}-1) * \mathrm{IL}-6$ was significant when comparing the control and stage 2 HTN group $(p<0.05)$, between stage 1 HTN and stage 2 HTN $(p<0.05)$ and between stage $2 \mathrm{HTN}$ and stage $2 \mathrm{HTN}+\mathrm{T} 2 \mathrm{DM}(p<0.05)$.

\section{Discussion}

Essential HTN is often associated with T2DM due to common pathophysiological pathways that include oxidative stress and inflammation. These increase the risk of morbidity and mortality due to CVD and renal disease. ${ }^{42}$ Changes in individual oxidative stress and inflammatory biomarkers in HTN and T2DM have been previously studied, ${ }^{8,43-44}$ but how or whether oxidative stress and inflammatory biomarkers act together in HTN progression with and without presence of T2DM has not been fully elucidated. Inflammatory and oxidative stress biomarkers were analyzed with a focus on comparing a control group, an HTN and an HTN+T2DM 
Table 3 Biomarker levels with respect to HTN stages with and without T2DM

\begin{tabular}{|c|c|c|c|c|c|}
\hline Category & Control & Stage I HTN & Stage 2 HTN & $\begin{array}{l}\text { T2DM + } \\
\text { stage I HTN }\end{array}$ & $\begin{array}{l}\text { T2DM + } \\
\text { stage } 2 \text { HTN }\end{array}$ \\
\hline \multirow[t]{2}{*}{ IGF-I (pg/mL) } & $303(533)^{\mathrm{b}}$ & $289(476)^{d}$ & 105 (188) & $249(220)$ & $263(57 I)$ \\
\hline & $n=53$ & $n=49$ & $\mathrm{n}=7$ & $\mathrm{n}=55$ & $n=9$ \\
\hline \multirow[t]{2}{*}{ MCP-I (pg/mL) } & $187(80)^{\mathrm{a}}$ & $172(106)^{c}$ & $280(457)^{e, f}$ & $204(134)$ & $193(158)$ \\
\hline & $\mathrm{n}=54$ & $n=48$ & $n=7$ & $\mathrm{n}=55$ & $n=9$ \\
\hline \multirow[t]{2}{*}{ (MCP-I/IGF-I)*IL-6 } & $7.3\left(19.0^{\mathrm{a}}\right.$ & $7.2(20.7)^{\mathrm{c,d}}$ & $54.2(96.4)^{f}$ & $16.3(4 \mid .7)$ & $7.1(5.9)$ \\
\hline & $\mathrm{n}=52$ & $n=48$ & $n=6$ & $n=42$ & $\mathrm{n}=8$ \\
\hline
\end{tabular}

Notes: aSignificant difference between control and stage 2 HTN group ( $p<0.05)$; bsignificant difference between control and T2DM with stage I HTN group ( $p<0.05)$; 'significant difference between stage I HTN and stage 2 HTN group $(p<0.05)$; ${ }^{d}$ significant difference between stage I HTN and T2DM with stage I HTN group ( $\left.p<0.05\right)$; esignificant difference between stage $2 \mathrm{HTN}$ and T2DM + stage I HTN group ( $p<0.05)$; 'significant difference between stage $2 \mathrm{HTN}$ and T2DM + stage $2 \mathrm{HTN}$ group $(p<0.05)$. Abbreviations: HTN, hypertension; IGF I, insulin-like growth factor; IL-I $\beta$, interleukin-I $\beta$; MCP-I, monocyte chemoattractant protein I; T2DM, type 2 diabetes mellitus.

group. A further category of biomarker levels with respect to stages 1 and 2 HTN was also included.

A significant increase in urinary 8-iso- $\mathrm{PGF}_{2} \alpha$ levels in the HTN+T2DM participants indicated the presence of lipid peroxidation due to oxidative stress. The increase in 8-iso- $\mathrm{PGF}_{2} \alpha$ levels between control and HTN, and HTN to HTN+T2DM participants, although not significant, is an important finding within the clinical context and shows a consistent increase in oxidative stress when HTN and T2DM are co-presenting. Previous work has shown that erythrocyte GSH significantly increased in association with diabetes progression, suggesting an increase in oxidative stress due to hyperglycemia, but in the current study a decrease in GSH occurred in the HTN+T2DM group when compared to the control and HTN groups. ${ }^{11}$ Our finding suggests that HTN in the presence of T2DM has compromised the de novo synthesis of GSH. Decreasing GSH and increasing 8-iso- $\mathrm{PGF}_{2} \alpha$ support the hypothesis that oxidative stress increases considerably in HTN especially in combination with diabetes. Therefore, possible clinical effects of HTN may also be detected earlier especially in TD2M patients, by focusing on changes in these emerging biomarkers of oxidative stress using 8-iso- $\mathrm{PGF}_{2} \alpha$ and erythrocyte GSH levels.

No significant changes in urinary 8-OHdG levels were observed when control, HTN, and HTN+T2DM groups were compared. In previous studies $8-\mathrm{OHdG}$ was observed to be higher in the prediabetic and hypertensive states in a homogeneous, non-medicated cohort. ${ }^{19,46}$ It should be noted that the current study aimed at investigating oxidative stress and inflammation in HTN when combined with diabetes as part of clinical practice, rather than elucidating pathophysiological pathways in disease progression and hence patients were kept on their prescribed medications. This may have influenced levels of 8-OHdG directly or as a consequence of TG, LDL-C, and blood glucose level not being at levels that lead to increases in 8-OHdG as has been suggested previously. ${ }^{10}$ This study therefore provides information, in part, on the efficacy of pharmacotherapy in treatment of HTN and when HTN is present together with T2DM. Apart from 8-OHdG, a substantial number of oxidative stress and inflammatory markers showed significant differences between the groups and especially between stages of HTN reflecting known pathophysiological changes and the difficulty in treating disease progression with a complex etiology.

The pro-inflammatory cytokines IL- $1 \beta$ and IL- 6 in patients with HTN did not increase in contrast to previous studies. ${ }^{12,26,47}$ Moreover, HTN patients seemed to have a decreased pro-inflammatory IL-1 $\beta$ level, which may be either due to statin use in this cohor ${ }^{48}$ or IL-10 activity, which was lower in the HTN group. ${ }^{49}$ While NSAID medication may contribute to HTN, ${ }^{50}$ the control group was not on antihypertensive medication. There was no significant difference in NSAID medication use between the HTN and HTN+T2DM groups, suggesting that the influence, if any, of NSAIDs is similar. Our study reflects the clinical findings associated with HTN in the presence of T2DM and suggests that T2DM does increase oxidative stress and inflammation in HTN patients when present, and requires careful monitoring to alleviate increased risk of further morbidity and mortality. In addition the T2DM group reported a significantly higher use of antihypertensives, which have antioxidative stress and antiinflammatory effects, yet there was a significant increase in oxidative stress and inflammation in the presence of T2DM. The significant difference between the control group and the HTN group for both IL-1 $\beta$ and IL-10 and for IL- $1 \beta$ and IL-10 in the HTN and the HTN+T2DM therefore indicate the potential use of these markers in clinical settings when investigating HTN and T2DM.

The decreased levels of IGF-1 present in the HTN+T2DM patients in our study cohort support previous findings of an 
association with IGF-1 and a higher risk of HTN+T2DM, ${ }^{33,51}$ suggesting IGF-1 may be a useful clinical marker. Only stage 2 HTN patients showed a significant increase in MCP-1 levels and an associated significant decrease in IGF-1. Interactions between these two biomarkers have also been observed in a previous study. ${ }^{52}$ Both IGF-1 and MCP-1 are biomarkers which indicate aggravation of inflammation in hypertensive patients. In relation to our findings of increased levels of MCP-1 in the stage 2 HTN group, in a more recent study associating increased levels of MCP-1 and the expression of platelet CD40 in hypertensive patients have been demonstrated $^{53}$ and further expands our understanding of the immune responses' contribution to the inflammatory and oxidative stress milieu when considering HTN and CVD. Medication controlling the levels of these biomarkers might be useful to prevent severe HTN from developing. The increased ratio of MCP-1 and IGF-1 with IL-6 in stage 2 hypertensive participants further highlights the interactions that occur between these biomarkers with disease progression. ${ }^{54,55}$ The current study has demonstrated that a decrease in MCP-1 is induced by IGF-1, and that decreasing IGF-1 is in turn induced by IL-6 associated with an increase in MCP-1. Indeed, IGF-1 is able to reduce inflammation by decreasing MCP-1 levels associated with lower recruitment of monocytes. ${ }^{54}$ However, pro-inflammatory cytokines such as IL-6 have an inhibitory effect on IGF-1 signaling pathways through blocking its receptor substrate..$^{55}$

Both oxidative stress and inflammation have been shown to be affected by HTN and T2DM disease progression especially when consideration is taken of HTN stages. This suggests stricter medication control especially in the HTN groups, which showed higher than optimal BP regardless of medication use.

\section{Disclosure}

The authors report no conflicts of interest in this work.

\section{References}

1. who.int [homepage on the Internet]. Raised blood pressure. Geneva: World Health Organization; 2008. Available from: http://www.who.int/ cardiovascular_diseases/en/. Accessed May 21, 2017.

2. Long AN, Dagogo-Jack S. The comorbidities of diabetes and hypertension: mechanisms and approach to target organ protection. $J$ Clin Hypertens (Greenwich). 2011;13(4):244-251.

3. Alder AI, Stratton IM, Neil HA, et al. Association of systolic blood pressure with macrovascular and microvascular complications of type 2 diabetes. BMJ. 2000;321(7258):412-419.

4. Yannoutsos A, Ahouah M, Tubiana CD, Topouchian J, Touboul C, Safar ME, Blacher J. Hemodynamic parameters in hypertensive diabetic patients. J Hypertens. 2016;34(6):1123-1131.

5. Ndisang JF, Vannacci A, Rastogi S. Oxidative stress and inflammation in obesity, diabetes, hypertension, and related cardiometabolic complications. Oxid Med Cell Longev. 2014;2014:506948.
6. Duarte DA, Silva KC, Rosales MA, Lopes de Faria JB, Lopes de Faria JM. The concomitance of hypertension and diabetes exacerbating retinopathy: the role of inflammation and oxidative stress. Curr Clin Pharmacol. 2013;8(4):266-277.

7. Sower JR, Epstein M. Diabetes mellitus and associated hypertension, vascular disease, and nephropathy. An update. Hypertension. 1995;26(6 Pt 1):869-879.

8. Cheung BM, Li C. Diabetes and hypertension: is there a common metabolic pathway ? Curr Atheroscler Rep. 2012;14(2):160-166.

9. Savini I, Catani MV, Evangelista D, Gasperi V, Avigliano L. Obesityassociated oxidative stress: strategies finalized to improve redox state. Int J Mol Sci. 2013;14(5):10497-10538.

10. Jelinek HF, Al-Aubaidy HA, Maschirow L, Meidinger S, Jamil DA, Butkowski E. Glutathione:Glutathione sulfide redox imbalance in early impaired fasting glucose. Cardiology and Angiology An International Journal. 2014;2(4):223-229.

11. Maschirow L, Khalaf K, Al-Aubaidy HA, Jelinek HF. Inflammation, coagulation, endothelial dysfunction and oxidative stress in prediabetes-Biomarkers as a possible tool for early disease detection for rural screening. Clin Biochem. 2015;48(9):581-585.

12. Butkowski EG, Brix LM, Al-Aubaidy HA, Kiat H, Jelinek HJ. Diabetes, oxidative stress and cardiovascular risk. J Med Clin Sci. 2016;5(1):17-23.

13. Nwose EU, Richards RS, McDonald S, Jelinek HF, Kerr RG, Tinley R. Assessment of diabetic macrovascular complications: a prediabetes model. Br J Biomed Sci. 2010;67(2):59-66.

14. Libby P, Simon DI. Inflammation and thrombosis: the clot thickens. Circulation. 2001;103(13):1718-1720.

15. Betteridge DJ. What is oxidative stress ? Metabolism. 2000;49(2 Suppl 1):3-8.

16. Rodrigo R, Prat H, Passalacqua W, Araya J, Guichard C, Bächler JP. Relationship between oxidative stress and essential hypertension. Hypertens Res. 2007;30(12):1159-1167.

17. Grossman E. Does increased oxidative stress cause hypertension? Diabetes Care. 2008;31 Suppl 2:S185-189.

18. Al-Aubaidy HA, Jelinek HF. 8-Hydroxy-2-deoxy-guanosine identifies oxidative DNA damage in a rural prediabetes cohort. Redox Rep. 2010;15(4):155-160.

19. Hinokio Y, Suzuki S, Hirai M, Suzuki C, Suzuki M, Toyota T. Urinary excretion of 8-oxo-7,8-dihydro-2'-deoxyguanosine as a predictor of the development of diabetic nephropathy. Diabetologia. 2002;45(6): 877-882.

20. Jelinek HF, Jamil DA, Al-Aubaidy HA. Impaired fasting glucose \& 8 -IsoProstaglandin F2 $\alpha$ in diabetes disease progression. Br J Med Med Res. 2014;4(33):5229-5237.

21. Davi G, Ciabattoni G, Consoli A, et al. In vivo formation of 8-isoprostaglandin F2alpha and platelet activation in diabetes mellitus: effects of improved metabolic control and vitamin E supplementation. Circulation. 1999;99(2):224-229.

22. Natarajan R, Lanting L, Gonzales N, Nadler J. Formation of an F2isoprostane in vascular smooth muscle cells by elevated glucose and growth factors. Am J Physiol. 1996;271(1 Pt 2):H159-H165.

23. Sueishi K, Ichikawa K, Kato K, Nakagawa K, Chen YX. Atherosclerosis: coagulation and fibrinolysis. Semin Thromb Hemost. 1998;24(3): 255-260.

24. Esmon CT. The interactions between inflammation and coagulation. $\mathrm{Br}$ J Haematol. 2005;131(4):417-430.

25. Frink RJ. Inflammatory atherosclerosis: characteristics of the injurious agent. Sacramento (CA): Heart Research Foundation; 2002.

26. Mojtaba E, Mahdi K, Kasbparast M, Amir S. Serum interleukin-1 beta plays an important role in insulin secretion in type II diabetic. International Journal of Biosciences. 2011;1(3):93-99.

27. Chamarthi B, Williams GH, Ricchiuti V, et al. Inflammation and hypertension: the interplay of interleukin-6, dietary sodium, and the renin-angiotensin system in humans. Am J Hypertens. 2011;24(10): $1143-1148$. 
28. Humbert M, Monti G, Brenot F, et al. Increased interleukin-1 and interleukin-6 serum concentrations in severe primary pulmonary hypertension. Am J Respir Crit Care Med. 1995;151(5):1628-1631.

29. Dinarello CA, Donath MY, Mandrup-Poulsen T. Role of IL-1beta in type 2 dabetes. Curr Opin Endocrinol Diabetes Obes. 2010;17(4):314-321.

30. van Exel E, Gussekloo J, de Craen AJ, Frölich M, Bootsma-van der Wiel A, Westendorp RG; Leiden 85 Plus Study. Low production capacity of interleukin-10 associates with the metabolic syndrome and type 2 diabetes: the Leiden 85-Plus Study. Diabetes. 2002;51(4):1088-1092.

31. Lima VV, Zemse SM, Chiao CW, Bomfim GF, Tostes RC, Clinton Webb $\mathrm{R}$, Giachini FR. Interleukin-10 limits increased blood pressure and vascular RhoA/Rho-kinase signaling in angiotensin II-infused mice. Life Sci. 2016;145:137-143.

32. Conti E, Pitocco D, Capoluongo E, Zuppi C, Ghirlanda G, Crea F, Andreotti F. IGF-1 and macrovascular complications of diabetes. Diabetes Care. 2003;26(5):1653-1654.

33. Teppala S, Shankar A. Association between serum IGF-1 and diabetes among U.S. adults. Diabetes Care. 2010;33:2257-2259.

34. Schutte AE, Volpe M, Tocci G, Conti E. Revisiting the relationship between blood pressure and insulin-like growth factor-1. Hypertension. 2014;63(5):1070-1077.

35. Panee J. Monocyte chemoattractant protein 1 (MCP-1) in obesity and diabetes. Cytokine. 2012;60(1):1-12.

36. Piemonti L, Calori G, Lattuada G, et al. Association between plasma monocyte chemoattractant protein-1 concentration and cardiovascular disease mortality in middle-aged diabetic and nondiabetic individuals. Diabetes Care. 2009;32(11):2105-2110.

37. American Diabetes Association. Diagnosis and classification of diabetes mellitus. Diabetes Care. 2010;33(1):S62-69.

38. Guide to management of hypertension 2008. Heart Foundation; 2010. Available from: https://www.heartfoundation.org.au/images/uploads/ publications/HypertensionGuidelines2008to2010Update.pdf. Accessed May 21, 2017.

39. Kjeldsen S, Feldman RD, Lisheng L, et al. Updated national and international hypertension guidelines: a review of current recommendations. Drugs. 2014;74(17):2033-2051.

40. Definition, diagnosis and classification of diabetes mellitus and its complications: report of a WHO consultation. Part 1: Diagnosis and classification of diabetes mellitus. Geneva: World Health Organization; 1999. Available from: http://apps.who.int/iris/handle/10665/66040. Accessed May 21, 2017.

41. McCormack T, Krause T, O’Flynn N. Management of hypertension in adults in primary care: NICE guideline. Br J Gen Pract. 2012;62(596): $163-164$.
42. Yan Q, Sun D, Li X, et al. Association of blood glucose level and hypertension in elderly Chinese subjects: a community based study. BMC Endocr Disord. 2016;16(1):40.

43. Savoia C, Schiffrin EL. Vascular inflammation in hypertension and diabetes: molecular mechanisms and therapeutic interventions. Clin Sci (Lond). 2007;112(7):375-384.

44. Al-Shukaili A, AL-Ghafri S, Al-Marhoobi S, Al-Abri S, Al-Lawati J, Al-Maskari M. Analysis of inflammatory mediators in type 2 diabetes patients. Int J Endocrinol. 2013;2013:976810.

45. Tiwari BK, Pandey KB, Abidi AB, Rizvi SI. Markers of oxidative stress during diabetes mellitus. J Biomark. 2013;2013:378790.

46. Broedbaek K, Weimann A, Stovgaard ES, Poulsen HE. Urinary 8-oxo7,8-dihydro-2'-deoxyguanosine as a biomarker in type 2 diabetes. Free Radic Biol Med. 2011;51(8):1473-1479.

47. Al-Daghri NM, Bindahman LS, Al-Attas OS, et al. Increased circulating ANG II and TNF-alpha represents important risk factors in obese saudi adults with hypertension irrespective of diabetic status and BMI. PloS One. 2012;7(12):e51255

48. Dinh QN, Drummond GR, Sobey CG, Chrissobolis S. Roles of inflammation, oxidative stress, and vascular dysfunction in hypertension. Biomed Res Int. 2014;2014:406960.

49. Kim HY, Cha HJ, Kim HS. CCL5 upregulates IL-10 expression and partially mediates the antihypertensive effects of IL-10 in the vascular smooth muscle cells of spontaneously hypertensive rats. Hypertens Res. 2015;38(10):666-674.

50. Johnson AG. NSAIDs and increased blood pressure. What is the clinical significance? Drug Saf. 1997;17(5):277-289.

51. Zhang L, Curhan GC, Forman JP. Plasma insulin-like growth factor-1 level and risk of incident hypertension in non-diabetic women. J Hypertens. 2011;29(2):229-235.

52. Sukhanov S, Snarski P, Higashi Y, Shai S, Delafontaine P. Insulin-like growth factor I (IGF-1) reduces chemokines and recruitment of monocytes into atherosclerotic plaque: potential mechanism mediating IGF-1 induced atheroprotection. FASEB J. 2015;29(1).

53. Stumpf C, Raaz D, Klinghammer L, Schneider M, Schmieder RE, Garlichs CD, Achebach S. Platelet CD40 contributes to enhanced monocyte chemoattractant protein 1 levels in patients with resistant hypertension. Eur J Clin Invest. 2016;46(6):564-571.

54. Arendt BK, Velaszquez-Dones A, Tschumper RC, Howell KG, Ansell SM, Witzig TT, Jelinek DF. Interleukin 6 induces monocyte chemoattractant protein-1 expression in myeloma cells. Leukemia. 2002;16(10):2142-2147.

55. Al-Shanti N, Stewart CE. Inhibitory effects of IL-6 on IGF-1 activity in skeletal myoblasts could be mediated by the activation of SOCS-3. J Cell Biochem. 2012;113(3):923-933.
Journal of Inflammation Research

\section{Publish your work in this journal}

The Journal of Inflammation Research is an international, peer-reviewed open access journal that welcomes laboratory and clinical findings on the molecular basis, cell biology and pharmacology of inflammation including original research, reviews, symposium reports, hypothesis formation and commentaries on: acute/chronic inflammation; mediators of

\section{Dovepress}

inflammation; cellular processes; molecular mechanisms; pharmacology and novel anti-inflammatory drugs; clinical conditions involving inflammation. The manuscript management system is completely online and includes a very quick and fair peer-review system. Visit http://www.dove press.com/testimonials.php to read real quotes from published authors. 